\begin{tabular}{|c|c|c|c|c|c|c|c|c|c|}
\hline Age & $<16$ & $<20$ & $20-29$ & $30-39$ & $40-49$ & 50-59 & 60-69 & $70-79$ & $80-89$ \\
\hline No of patients & 3 & 1 & 1 & 4 & 7 & 4 & 3 & 2 & 1 \\
\hline Doxy & & & & 1 & & & & & \\
\hline Cipro & 1 & & & 1 & 1 & & & & \\
\hline Doxy/cipro & & & & 1 & 3 & 1 & & 1 & \\
\hline Gent & & & & & & & 1 & & \\
\hline Gent/cipro & & & & & & & 1 & & \\
\hline \multicolumn{10}{|l|}{ Gent/Cipro } \\
\hline Ceftriax & & & & 1 & & & & & \\
\hline Gent/cipro/Doxy & & & & & & 1 & & & \\
\hline Taz/Doxy & 1 & & & & & & & 1 & \\
\hline Taz/Cipro & & & & & & 1 & & & \\
\hline Taz & & & & & 1 & & & & \\
\hline Taz/Flucon & & & & & 1 & & & & \\
\hline Augmentin & & 1 & & & 1 & & 1 & & \\
\hline Cephalexin & & & & & & 1 & & & \\
\hline $\begin{array}{l}\text { Ceftriaxone/ } \\
\text { doxy }\end{array}$ & & & 1 & & & & & & \\
\hline \multicolumn{10}{|l|}{ Ceftriaxone/ } \\
\hline Cipro/doxy & & & & & 1 & & & & \\
\hline Doxy/Cipro/Mero & & & & & & & & & 1 \\
\hline
\end{tabular}

\section{P182 CLINICAL CASE NOTE REVIEW: ARE ALL APPROPRIATE UNDER- 16S BEING TESTED FOR CHLAMYDIA AND GONORRHOEA?}

Carly Stevenson*. University of Glasgow, Glasgow, UK

10.1136/sextrans-2015-052126.225

Background/introduction The number of young people under the age of 16 diagnosed with Chlamydia Trachomatis and Neisseria Gonorrhoea continues to rise annually. STI testing is essential to promote safe sexual practice, minimise stigma and monitor levels of anti-microbial resistance.

Aim(s)/objectives This project aimed to determine the percentage of under- $16 \mathrm{~s}$ who were not tested for chlamydia and gonorrhoea between January and April 2013. The role of testing was established with aims including:

- Comparison of attendance, testing and infection rates of males and females.

- Analysis of documented reasons for not testing.

- Percentage of eligible patients not documented to have been offered a sexual health screen (SHS).

Methods 200 patients were randomly selected by attendance at sexual health clinics over a four month period. Information was then gathered on each consultation using $\mathrm{NaSH}$ software. Information gathered included gender, age at consultation, tests requested and clinic attended.

Results Results showed that $56 \%$ of patients were not tested for chlamydia and gonorrhoea; $6 \%$ were not documented as having been offered a SHS. Reasons for not testing are documented below in descending order of prevalence:

\begin{tabular}{ll}
\hline Reason & Untested population (\%) \\
\hline SHS up to date & 37 \\
SHS declined & 18 \\
SHS not indicated & 14 \\
First time sexual activity & 12 \\
Not sexually active & 10 \\
Too early for SHS & 4 \\
\hline
\end{tabular}

Discussion/conclusion The rate of failure to document the offer of a SHS is reassuringly low. Rates of attendance and infection were highest in females. Healthcare professionals should continue to encourage testing of the sexually active and ensure offers of SHS are documented.

\section{P183 TRICHLOROACEITIC ACID (TCA) - A FORGOTTEN TREATMENT FOR GENITAL WARTS?}

Laura Clarke*, Rak Nandwani. Sandyford Initiative, Glasgow, UK

\subsection{6/sextrans-2015-052126.226}

Background/introduction Genital warts (GWs), are the most common STI in the UK. They can have a huge psychological impact on patients and can be very difficult to clear. There has been little research and few RCTs comparing treatments. In Glasgow, TCA is reserved for patients that standard treatments have failed.

Aim(s)/objectives To describe the use of TCA as a treatment for persistent and recurrent GWs and to review the local practice and protocol.

Methods We conducted a retrospective case review of all patients who received TCA in 2013 in our integrated sexual and reproductive health service with follow-up to the end of 2014. Patients were identified by prescriptions of TCA on our electronic patient record.

Results TCA was used on all types of warts in a variety of multiple locations. 20 out of 27 patients achieved clearance with TCA in 2013 (74\%) and of these, 5 experienced recurrence in 2014 (25\%). Patients with some level of immunosuppression may benefit from TCA treatment and respond earlier than those with a fully functioning immune system.

Discussion/conclusion TCA is an effective treatment for persistent and recurrent GWs; either used alone or with an adjuvant therapy, with relatively few side-effects. It can provide patients who have exhausted many/all other treatment options, positive results and improve mental well-being.

This audit also highlights the importance of improved documentation of warts by our staff and closer adherence to the existing clinic protocol for the management of GWs.

\section{P184 EXPERIENCE OF THE TENDER PROCESS AND INTEGRATION OF SEXUAL HEALTH SERVICES: STAFF SURVEY}

Cara Saxon*. Bridgewater Community Healthcare NHS Foundation Trust, Manchester, UK 10.1136/sextrans-2015-052126.227

Background/introduction In September 2013 four services merged to form a new integrated sexual health (ISH) service under a new NHS provider following a tender process.

Aim(s)/objectives To ascertain staff experience of the tender process and integration of sexual health services.

Methods All staff were asked to complete an online survey in 01/2015 (via SurveyMonkey ${ }^{\circledR}$ ). Staff who did not transfer to the new NHS provider or who left the service before 01/2015 were not included.

Results 23/38 (61\%) staff members (including medical, nursing, administrative and allied health professionals) responded. 5/23 $(22 \%)$ were entirely/predominantly from a genitorurinary background and 9/23 (39\%) entirely/predominantly contraception 
background. 16/23 (70\%) worked at one of the four previous services. Staff experience of the tender/integration process in terms of 'stress'/'excitement' levels are reported in the Table 1.

\begin{tabular}{|c|c|c|c|c|}
\hline & \multicolumn{4}{|l|}{ Pre } \\
\hline & $\begin{array}{l}09 / 2013 \\
(\%)\end{array}$ & $\begin{array}{l}\text { Months } \\
0-6(\%)\end{array}$ & $\begin{array}{l}\text { Months } \\
7-12(\%)\end{array}$ & $\begin{array}{l}\text { Month } 12 \\
\text { onwards (\% }\end{array}$ \\
\hline Moderate-Very Exciting & $0(0)$ & $1(5)$ & $3(15)$ & $11(50)$ \\
\hline Mildly exciting & $0(0)$ & $0(0)$ & $4(20)$ & $2(9)$ \\
\hline No different & $4(27)$ & $0(0)$ & $2(10)$ & $0(0)$ \\
\hline Mildly stressful & $3(20)$ & $2(10)$ & $3(15)$ & $4(18)$ \\
\hline Moderate-Very Stressful & $9(60)$ & $17(85)$ & $13(65)$ & $7(32)$ \\
\hline Total respondents* & 15 & 20 & 20 & 22 \\
\hline
\end{tabular}

$14 / 22(64 \%)$ of staff believe that SH services should be integrated. 17/22 (77\%) feel patients are now getting a better service (with further improvements needed).

Themes Main 'positives experienced': new skills gained, increasing integration/offer of a 'one-stop-shop' service. Main 'challenges experienced': resistance to change, clash of specialty 'cultures'. The predominant 'suggestion for improvement' was better communication with all staff throughout the process.

Discussion/conclusion The experience of the tender process and early months in the new ISH service was stressful for many staff. This improved with time and staff reported feeling increasingly excited about the new service. Better communication from commissioners and service providers to all staff involved may improve the overall experience of those going through the process in the future.

\section{P185 USING THE STIF PORTFOLIO IN AN "INTEGRATION" TRAINING STRATEGY}

Lee Portas, Ruth Taylor, Ashini Fox*. Nottingham University Hospitals Trust, Nottingham, UK

\subsection{6/sextrans-2015-052126.228}

Background Many UK sexual health clinics are in the process of integrating Sexual and Reproductive Health (SRH) and GUMedicine (GUM) services. Amongst the many challenges they face is that of appropriately training newly integrated staff. Our unit is has recently undergone integration of contraception, termination, outreach and GUM/HIV services. Central to this process was the establishment of a comprehensive training strategy for all clinical staff.

Objectives To describe the successful implementation of an integration training strategy using BASHH's STIF portfolio between 2012-2014.

Methods An initial baseline staff survey demonstrated a lack of consistency of formal sexual health qualifications amongst both SRH and GUM staff. It also highlighted considerable skills amongst some HCAs who had lacked opportunity to formalise them. Our desire was to use existing national qualifications and provide equality of access to all grades of staff.

Results Between 2012-2014 we ran 2 STIF theory courses and 4 STIFLevel 1 assessments. In total 53 staff attended STIF theory and 45 successfully completed STIFLevel 1 (including 8 HCAs). A further 7 senior nurses and $2 \mathrm{SRH}$ doctors have completed STIFIntermediate. One band 7 GUM nurse has also completed STIFAdvanced.

Conclusion The STIF portfolio has provided practical and effective tools in training and assessing staff during our local integration process. We believe that the existence of a clear training strategy helped maintain moral and staff retention during a potentially difficult time and the high level of national qualification amongst our staff will hopefully stand us in good stead in the current commissioning climate.

\section{P186.1 DOES CHLAMYDIA TESTING IN GENERAL PRACTICE MEAN MISSED OPPORTUNITIES FOR THE DIAGNOSIS OF OTHER STIS?: A COMPARISON OF THE POPULATION TESTED IN GENERAL PRACTICE VERSUS SEXUAL HEALTH CLINICS IN BRITAIN}

${ }^{1}$ Soazig Clifton*, ${ }^{1}$ Catherine Mercer, ${ }^{2}$ Jackie Cassell, ${ }^{1}$ Pam Sonnenberg, ${ }^{3}$ Michelle Lu, ${ }^{1,4}$ Sarah Woodhall, ${ }^{4}$ Kate Soldan. ${ }^{1}$ University College London, London, UK; ${ }^{2}$ Brighton and Sussex Medical School, Brighton, UK; ${ }^{3}$ University of Manchester, Manchester, UK; ${ }^{4}$ Public Health England, London, UK

\subsection{6/sextrans-2015-052126.229}

Background Chlamydia testing in general practice may provide opportunities to diagnose those who do not attend sexual health (GUM) clinics. However, as comprehensive STI testing is less likely to be offered in general practice, opportunities could be missed to test, diagnose and treat other STIs including HIV if people at higher sexual risk test in general practice.

Aim To compare demographic, behavioural, and HIV testing characteristics of those tested for chlamydia in general practice with those tested in GUM.

Methods A probability sample survey of the British population undertaken 2010-2012. We analysed weighted data on individuals aged 16-44, reporting at least one sexual partner ever, who reported a chlamydia test in the past year $(\mathrm{n}=1583)$.

Results 26.0\% (24.7-27.4) of women and 16.1\% (14.9-17.3) of men reported testing for chlamydia in the past year, of whom $41.4 \%(38.6-44.2)$ of women and 20.5\% (17.4-24.0) of men tested in general practice. Women tested in general practice were more likely to be older, in a relationship, and to live in rural areas. Men and women tested in general practice reported lower STI risk in terms of (past 5 years): partner numbers, same-sex partners, and overlapping partnerships. Those tested in general practice were less likely to report an HIV test (past 5 years).

Discussion/conclusion While those tested for chlamydia in general practice generally reported lower risk behaviours, rural populations were over-represented, and HIV testing was lower. Pathways to comprehensive STI care need to be universally available for higher risk individuals.

\section{P186 HIGH LEVELS OF USE OF RECREATIONAL DRUGS AND ALCOHOL WITHIN AN INNER LONDON SEXUAL HEALTH CLINIC}

Rosemarie Turner*, Neil Turner, Ann Sullivan, Sara Day. Chelsea and Westminster Hospital, London, UK

\subsection{6/sextrans-2015-052126.230}

Background Drug and alcohol use by patients attending sexual health clinics is not widely assessed as routine. BASHH history taking guidelines and position statement on recreational drug use 